\title{
Radio haloes of star-forming galaxies
}

\author{
Aditi Vijayan ${ }^{\circledR},{ }^{1,2 \star}$ Biman B. Nath, ${ }^{1}$ Prateek Sharma ${ }^{\odot 2}$ and Yuri Shchekinov ${ }^{1,3}$ \\ ${ }^{1}$ Raman Research Institute, Bangalore 500080, India \\ ${ }^{2}$ Joint Astronomy Programme and Department of Physics, Indian Institute of Science, Bangalore 560012, India \\ ${ }^{3}$ Lebedev Physical Institute of Russian Academy of Sciences, ASC, Moscow 117997, Russia
}

Accepted 2019 December 13. Received 2019 December 11; in original form 2019 July 30

\begin{abstract}
We study the synchrotron radio emission from extra-planar regions of star-forming galaxies. We use ideal magnetohydrodynamic simulations of a rotating Milky Way-type disc galaxy with distributed star formation sites for three star formation rates $\left(0.3,3,30 \mathrm{M}_{\odot} \mathrm{yr}^{-1}\right)$. From our simulations, we see emergence of galactic-scale magnetized outflows, carrying gas from the disc. We compare the morphology of the outflowing gas with hydrodynamic simulations. We look at the spatial distribution of magnetic field in the outflows. Assuming that a certain fraction of gas energy density is converted into cosmic ray energy density, and using information about the magnetic field, we obtain synchrotron emissivity throughout the simulation domain. We generate the surface brightness maps at $1.4 \mathrm{GHz}$. The outflows are more extended in the vertical direction than radial and hence have an oblate shape. We further find that the matter right behind the outer shock shines brighter in these maps than that above or below. To understand whether this feature can be observed, we produce vertical intensity profiles. We convolve the vertical intensity profile with the typical beam sizes of radio telescopes, for a galaxy located at $10 \mathrm{Mpc}$ to estimate the radio scale height and compare with observations. The radio scale height is $\sim 300-1200 \mathrm{pc}$, depending on the resolution of the telescope. We relate the advection speed of the outer shock with the surface density of star formation as $\mathrm{V}_{\mathrm{adv}} \propto \Sigma_{\mathrm{SFR}}^{0.3}$, which is consistent with earlier observations and analytical estimates.
\end{abstract}

Key words: galaxies: evolution-galaxies: haloes - galaxies: spiral-radio continuum: galaxies.

\section{INTRODUCTION}

Morphology of galaxies in different wavebands provide us with clues to various physical processes occurring in a galaxy, and therefore helps us to understand different aspects of galactic evolution. The physical processes such as outflows, exchange of mass and momentum between the star-forming disc and the circumgalactic medium, unleashed by star formation activity in a galaxy have far reaching consequences, ranging from regulation of star formation rate (SFR) in a galaxy (Krumholz et al. 2012; Lim et al. 2018; Dekel et al. 2019) to influencing the physical state of the circumgalactic medium (Tumlinson et al. 2017) and enrichment of the intergalactic medium (e.g. Oppenheimer \& Davé 2006). Since these physical processes are complex in nature and are mediated by multiple factors (e.g. interaction between various gas phases, cooling), it is important to compare theoretical predictions with observations in different wavebands in order to ensure the validity of stellar feedback models. In this regard, results of hydrodynamic simulations have been

\footnotetext{
^E-mail: aditiv@rri.res.in
}

compared with optical (Cooper et al. 2008), X-ray (Suchkov et al. 1996; Cooper et al. 2009; Vijayan et al. 2018), and molecular lines (Roy et al. 2016).

Radio emission from star formation in galaxies has long been an important field of study (Condon 1992). Synchrotron emission produced by cosmic ray electrons (CREs), accelerated by supernovae, dominates the radio luminosity $(v<10 \mathrm{GHz})$ of star-forming galaxies (remaining small fraction arising from free-free emission in $\mathrm{H}$ II regions). Observations of edge-on spiral galaxies have shown that the synchrotron emission can reach vertical heights of several kpc (Hummel \& van del Hulst 1989; Hummel et al. 1991; Dahlem et al. 1995, 2006; Irwin et al. 1999, 2012). The vertical extent of the radio halo, referred to as the radio scale height, depends on the magnetic field configuration and cosmic ray (CR) transport properties. It is smaller than the magnetic scale height (Beck 2009). The radio scale height is also related to advection of CRs, and therefore, possibly related to the star formation process, which may dictate the convective speed. CRs, thus, can potentially trace galactic outflows, and the study of radio haloes is important in the overall understanding of physics of outflows. 
The morphology of radio haloes of star-forming galaxies has become important from another quarter as well. Recent balloon measurements with ARCADE-2 have shown the existence of a bright Galactic high-latitude diffuse radio emission, referred to as the monopole (Fixsen et al. 2011; Kogut et al. 2011), confirming early tentative measurements (e.g. Costain 1960). The magnitude of the monopole crucially depends on the assumed radio halo morphology of our Galaxy. Explaining this excess brightness (with brightness temperature of order $\sim 1 \mathrm{~K}$ at $1.4 \mathrm{GHz}$ ) with an extragalactic component is difficult because the excess does not have a far-infrared (FIR) counterpart as predicted by the observed strong radio/FIR correlation in galaxies. Biermann et al. (2014) have invoked models of emission from shocks due to primordial massive stars at cosmological distances to understand this excess. To avoid such models, Subrahmanyan \& Cowsik (2013) tried to explain the emission by invoking a spherical radio halo of our Galaxy extending up to $\sim 15 \mathrm{kpc}$. But this has been doubted by Singal et al. (2015) by calling attention to the fact that observed radio morphology of edge-on galaxies do not show such a spherical geometry.

Observations of radio haloes of edge-on galaxies give a number of clues to their connection with star formation activity in the disc. For example, Dahlem et al. (1995) used observation of five edge-on galaxies to establish that a critical SFR surface density $\left(\dot{E}_{A}>10^{-4}\right.$ erg s $\mathrm{s}^{-1} \mathrm{~cm}^{-2}$ ) is required to support an extended halo. Assuming a Kroupa initial mass function, $10^{51}$ erg per supernovae and that a fraction $\sim 0.3$ thermalization of energy deposited, translates into an SFR surface density of $\sim 10^{-2} \mathrm{M}_{\odot} \mathrm{yr}^{-1} \mathrm{kpc}^{-2}$. Incidentally, this threshold is an order of magnitude lower than that of launching a galactic outflow (Lehnert \& Heckman 1996). Heesen et al. (2016) found that radio haloes exist for galaxies with an SFR density of $\geq 10^{-3} \mathrm{M}_{\odot} \mathrm{yr}^{-1} \mathrm{kpc}^{-2}$. Roy et al. (2013) and Vasiliev et al. (2019) explain these thresholds with the help of hydrodynamic simulations. An explicit connection to the radio halo would require information about the galactic magnetic field.

Dahlem et al. (1995) found the morphology of radio haloes to be oval-shaped, with the radial extent of the halo at the radio scale height being somewhat smaller than that along the mid-plane of the disc. The radial extent of the halo in the disc plane was further found to be larger (by a few kpc, on an average) than that of star formation. They explained this difference by invoking CR diffusion, with a value of diffusion coefficient $D \sim 10^{28}-10^{29} \mathrm{~cm}^{2} \mathrm{~s}^{-1}$, consistent with CR phenomenology. Interestingly, Dahlem et al. (2006) found the radial extent at a height to be comparable to the star formation extent along the disc. Clearly, the extra-planar radio emission is related to the distribution of star formation sites in the disc. This has been confirmed by Krause et al. (2018), who observed a sample of 13 edge-on spiral galaxies and found that the radio scale height depends on the extent of radio emission along the plane, but not on SFR. The SFR surface density in their sample of galaxies was low $\left(\leq 7 \times 10^{-3} \mathrm{M}_{\odot} \mathrm{yr}^{-1} \mathrm{kpc}^{-2}\right)$.

Due to uncertainties in obtaining the SFR in Dahlem et al. (1995), the correlation between extent of radio haloes and SFR was not replicated by Irwin et al. (1999). Irwin et al. (1999) attribute this to difference in time-scales over which star formation $\left(\sim 10^{7} \mathrm{yr}\right)$ takes place and that over which radio haloes can persist $\left[\sim 10^{8}\right.$ $\mathrm{yr}$, because of CRE lifetime, which is given by $\sim 0.8 \times 10^{8} \mathrm{yr}$ $\left.(v / 1.4 \mathrm{GHz})^{-0.5}(B / \mu \mathrm{G})^{-1.5}\right]$. Further, Irwin et al. (1999) observed discrete features above the star-forming regions in the disc characterized by spectral flattening, which they ascribe to winds launched by star formation process. In their spectral maps of galaxies, they noticed a steepening of the spectral index (i.e. it becomes more negative) away from the plane of the disc along the minor axis.
They explained this by arguing that CREs that populate the halo would have lost energy in the disc before being transported to the halo (Duric et al. 1998).

There are two time-scales involved here: (1) the time-scale of CR transport to extra-planar heights, which can occur either through diffusion or advection in case of an outflow, and (2) the radiation loss time-scale of electrons. As mentioned above, the radiation loss time-scale of electrons radiating at $1.4 \mathrm{GHz}$ is of the order of $\sim 80 \mathrm{Myr}$ for $\mu \mathrm{G}$ magnetic field. In comparison, the diffusion time-scale is $l^{2} / 2 D \sim 180 \mathrm{Myr}$ for a height of $3 \mathrm{kpc}$ and diffusion coefficient of $10^{28} \mathrm{~cm}^{2} \mathrm{~s}^{-1}$. Advection time-scale to reach the same height would be of the order of $\sim 30 \mathrm{Myr}$ for a wind speed of $100 \mathrm{~km} \mathrm{~s}^{-1}$. Therefore, a spectral steepening with height would correspond to the case of large magnetic field (short cooling timescale) and low wind speed. Confirming this, Duric et al. (1998) mentioned that morphology of radio haloes with spectral flattening at extra-planar regions did indicate the presence of winds, and had 'chimneys' features through which disc material had broken out. This was confirmed by high-resolution observations of Irwin et al. (2000), with the presence of discrete features in the radio haloes.

These ideas were confirmed by Heesen et al. (2016), who used one-dimensional (1D) transport model to understand how advection and diffusion modify the spectral index. They also found that while advection causes the spectral index to steepen gradually in a diffusion dominated scenario the spectral index hardly steepens within one scale height and steepens rapidly at larger heights. Recently, Heesen et al. (2018) used 1D CR transport equations, of advection and diffusion, in order to model the vertical intensity and spectral profile, fitting to radio continuum maps of 12 nearby galaxies. They found that an advection speed scaling, $\mathrm{v}_{\mathrm{adv}} \sim \Sigma_{\mathrm{SFR}}^{0.3}$, can explain the profiles. In the study by Krause et al. (2018), it was inferred that convection plays an important role in transporting CRs to extra-planar heights.

In this paper, we address some issues regarding the morphology, vertical intensity profile of radio haloes, such as its dependence on SFR surface density, for Milky Way-type galaxies with magnetohydrodynamic (MHD) simulations. Earlier simulations have looked at the dynamical and kinematical role CRs play in galaxy evolution (Booth et al. 2013; Pakmor et al. 2014; Salem \& Bryan 2014; Ruszkowski et al. 2017). These simulations attempt to understand how CR pressure can affect large outflow structure. Our focus is on the role of galactic winds in the determining the structure of extra-planar magnetic field and the synchrotron emission profiles of edge-on star-forming galaxies. The observations of Dahlem et al. (1995) showed that the radial extent of the radio halo at some height above the disc remains the same as the radial extent along the disc. Therefore, a disc-wide distribution of star formation sites is required to study the morphology. Our previous work on the effect of distributed star formation on the X-ray morphology (Vijayan et al. 2018) provides us with a suitable set-up for this study, when magnetic field is added.

\section{SET-UP}

\subsection{Disc and halo}

The set-up is identical to the three-dimensional (3D) set-up used in Vijayan et al. (2018). We simulate a Milky Way mass $\left(M_{\mathrm{vir}}=10^{12}\right.$ $\mathrm{M}_{\odot}$ ) galaxy that comprises a high-density, low-temperature rotating stellar disc $\left(M_{\text {disc }}=10^{10} \mathrm{M}_{\odot}\right)$ in equilibrium with a static halo. The halo gas is hotter $\left(T_{\text {vir }} \sim 3 \times 10^{6} \mathrm{~K}\right)$ and less dense than the $4 \times 10^{4}$ $\mathrm{K}$ disc. The initial density and pressure profiles are determined by 
solving the equations of hydrodynamic equilibrium between the rotating disc and the stationary halo. Mid-plane density is $3 \mathrm{~m}_{p}$ $\mathrm{cm}^{-3}$. The stellar disc is modelled by Miyamoto-Nagai potential and NFW profile is used for the dark matter potential. For further details on the set-up, including the density profiles, the reader is directed to section 2 of Vijayan et al. (2018).

\subsection{Initial magnetic field}

The magnetic field in the disc of our Galaxy is not very well understood. However, radio observations have been used to infer the probable topology and magnitude of the magnetic field. Sun et al. (2008) describe a functional form of the Galactic magnetic field, in the disc as well as in the halo (their equation 7), which we adopt here:

$$
B_{\phi}=B_{0} \exp \left[-\frac{R}{R_{0}}-\frac{|z|}{z_{0}}\right],
$$$$
B_{z}=B_{R}=0 .
$$

Here, $B_{0}$ is $2 \mu \mathrm{G}$ and $z_{0}$ and $R_{0}$ are equal to $400 \mathrm{pc}$ and $1.5 \mathrm{kpc}$, respectively (Sun et al. 2008). We have chosen the magnetic scale height, $z_{0}$, to be equal to the parameter $b$ of the disc potential. We have changed the configuration from Sun et al. (2008) in order to apply it to our simulated galaxy. While they had a constant magnetic field strength inside of a region $5.3 \mathrm{kpc}$ around the Galactic centre and follow the exponential form outside (scaling as $\exp [-(R-$ $\left.\left.\mathrm{R}_{\odot}\right) / R_{0}\right]$ ), we have made it exponential from the central region. This is because in our simulated galaxy, the peak of star formation sites is at a galactocentric radius of $\sim 1 \mathrm{kpc}$ (while for our Galaxy, it is around $\sim 4 \mathrm{kpc}$ ), and so the radial extent of our simulated galaxy is smaller than Milky Way.

The initial magnetic field has been set-up only in the disc region in using a vector potential. Since the thermal pressure is much higher than the magnetic pressure, the unbalanced Lorentz force leads to only a marginal ( $<10$ per cent) change in the density distribution in the disc for the period of interest, $10 \mathrm{Myr}$.

With an exponential decay of magnetic field strength beyond the disc we most certainly underestimate the magnetic field strength on the halo scale, but it is acceptable for our purposes because our focus is on the brightest radio emission concentrated close to the disc. Since we run simulations only over a starburst time-scale, which is much shorter than the expected dynamo time-scale, the magnetic field strength is simply the result of our initial conditions. Moreover, we are also missing the small-scale dynamo which is expected to be operating in the diffuse galactic halo because of turbulence.

\subsection{Numerical set-up}

We use a $[-6,6]^{3} \mathrm{kpc}^{3}$ Cartesian grid which is uniform in all the three directions with a resolution of $36 \mathrm{pc}$. We conduct all our simulations using the finite-volume code, PLUTO (Mignone et al. 2007). We use HLLD (for MHD runs) and HLLC (for HD runs) solvers with linear reconstruction for all simulations discussed here. Constrained transport has been used to ensure $\boldsymbol{\nabla} \cdot \boldsymbol{B}=0$ throughout. Outflow boundary conditions are used everywhere.

The energy and mass injection rate have been chosen so that the total SFR across the disc is $0.3,3$, and $30 \mathrm{M}_{\odot} \mathrm{yr}^{-1}$. We can estimate the mechanical energy and mass injection rate from the SFR by assuming Kroupa initial mass function and a factor of $\sim 0.3$ of the total deposited energy to be thermalized (Strickland \& Heckman 2007; Vasiliev et al. 2019) as

$L_{\text {mech }} \approx 10^{41} \frac{\operatorname{erg}}{\mathrm{s}}\left(\frac{\mathrm{SFR}}{\mathrm{M}_{\odot} \mathrm{yr}^{-1}}\right)$.

Supernova explosions and stellar winds also add mass to the surrounding interstellar medium, the precise amount of which is uncertain. We adopt a mass injection rate that is related to the SFR in the following way:

$\dot{M}_{\text {inj }}=0.1$ SFR.

We choose 1000 points in the disc for mass and energy injection to simulate the locations of $\mathrm{OB}$ associations, and for simplicity, all have identical mass and energy injection rates. The location of these points have been chosen to follow the Kennicutt-Schmidt law between the surface density of gas and surface density of star formation $\left(\Sigma_{\mathrm{SFR}} \propto \Sigma_{\text {gas }}^{1.4}\right)$. In the $z$-direction, the points have been distributed to follow the gas density. For an exponential gas distribution in the disc, this implies a radial distribution of injection points with a maximum at $\sim 1 \mathrm{kpc}$ for the parameters we have chosen (for details see appendix A of Vijayan et al. 2018).

\section{RESULTS}

We conduct 3D simulations using the above described set-up. We compare the density snapshots for MHD and HD runs for our fiducial run with $\mathrm{SFR}=3 \mathrm{M}_{\odot} \mathrm{yr}^{-1}$. In Fig. 1, we show the density slices in the $x=0$ plane for MHD (left) and HD (right) runs after 5 Myr of evolution. Star formation in each injection region creates a bubble of hot, low-density gas around it. Over time, bubbles from different injection regions merge and ultimately break out of the disc of the galaxy. The outflow generated is fastest close to the centre of the galaxy because it has the highest density and a higher number of injection points. In the outer regions of the disc, the shock is not strong enough due to fewer injection points. Since the initial conditions, including distribution of injection points, is symmetric along the $z$-axis, the outflow is similar in $z>0$ and $z<0$. Morphologies of MHD and HD runs are very similar, which is expected since the magnetic energy density is smaller than the thermal energy density. As injected gas rises, it lifts up clumps of dense disc gas. These clumps can be clearly seen as patches of high density surrounded by a low-density gas. As they move up, this dense disc gas gets mixed with the hotter injected gas.

Magnetic field is entrained in disc gas, as it traverses through the potential of galaxy. In Fig. 2, we show the magnitude of magnetic field $\left(|B|=\sqrt{B_{x}^{2}+B_{y}^{2}+B_{z}^{2}}\right)$ in the $x=0$ plane (left-hand panel) at $t=0$ (left-hand panel) and at $t=5 \mathrm{Myr}$ (right-hand panel) for the fiducial run. We can see that the disc region still contains substantial magnetic field. However, in the regions above and below the disc its distribution is highly non-uniform. There are regions inside of the outer shock that are devoid of magnetic field (e.g. at $y \simeq 2$ $3 \mathrm{kpc}$ ). The corresponding region in the density slice shows a drop in density. Thus, the magnetic field is preferentially high in regions of high density.

To further explore the spatial correlation between magnetic field and density, the left two panels of Fig. 3 show $|B|$ and the disc gas tracer for the first quadrant of the simulation in the $x=0$ plane. Tracers are scalar quantities that get advected with the fluid. We have used three kinds of tracers - for metallicity, disc gas, 


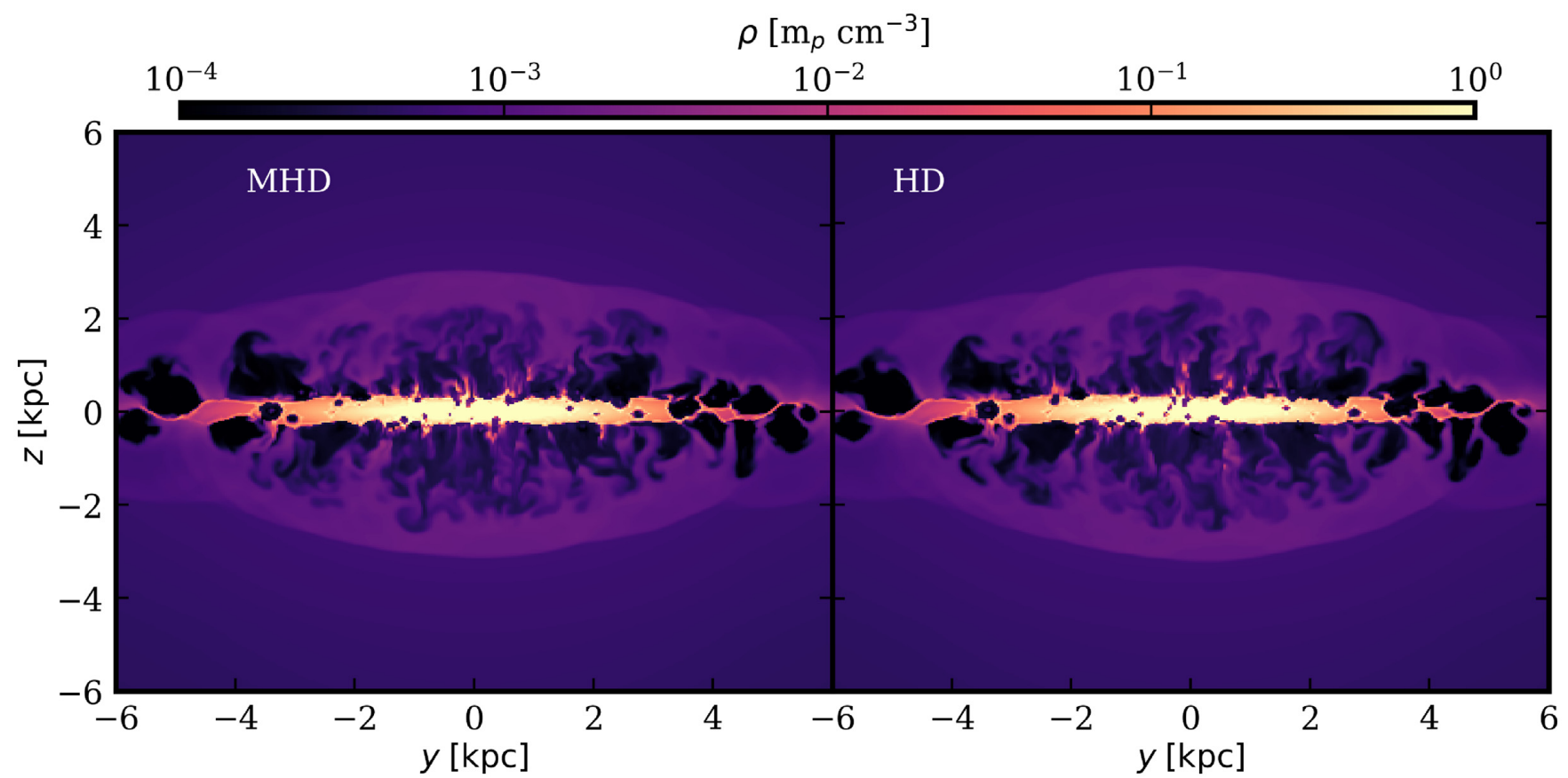

Figure 1. Density snapshots in the $x=0$ plane for MHD (left) and HD (right) runs at $5 \mathrm{Myr}$. Both the runs have identical initial conditions, resolutions, and location of injection points. The SFR is $3 \mathrm{M}_{\odot} \mathrm{yr}^{-1}$. Since the magnetic energy density is low these runs are very similar in morphology.

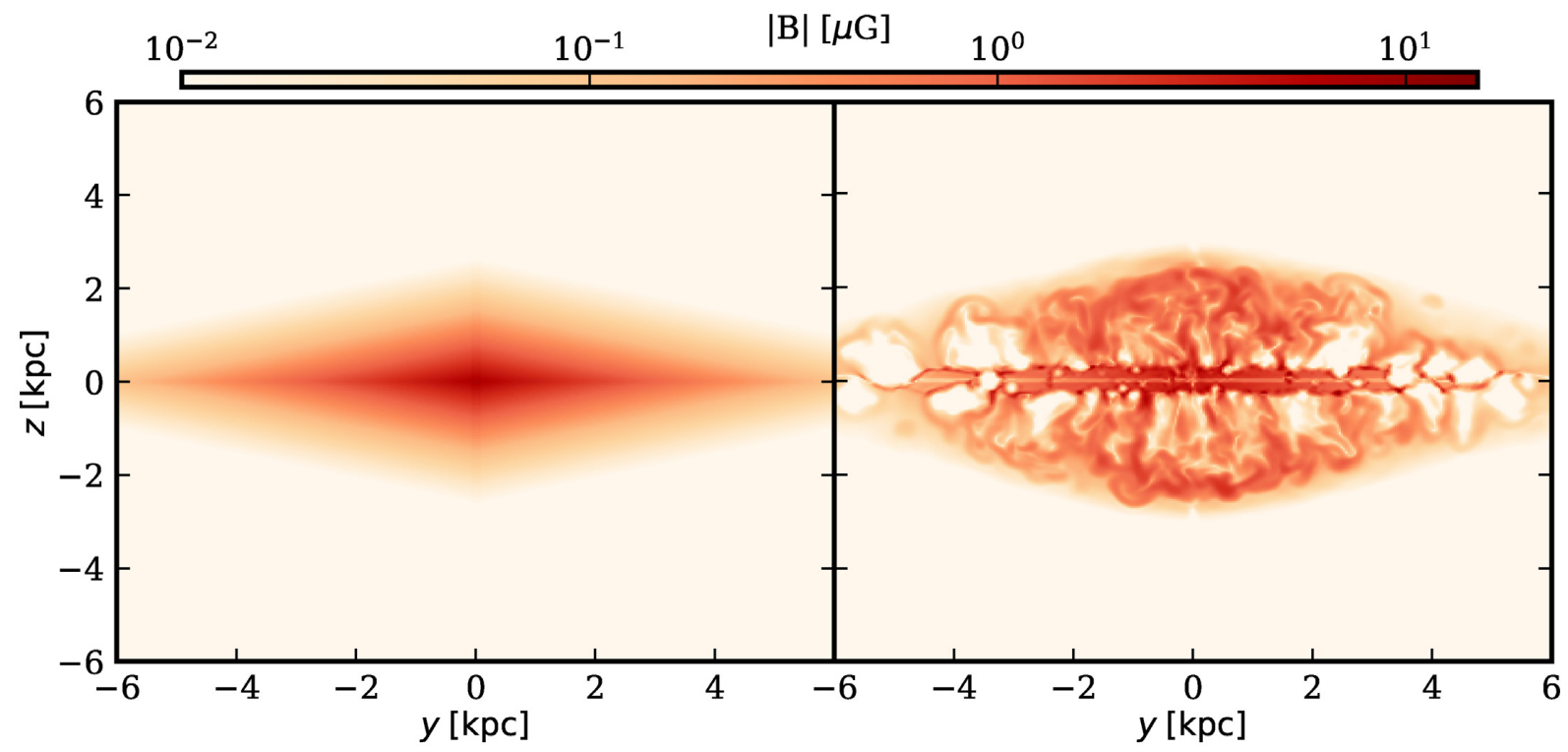

Figure 2. Magnetic field strength $\left(|B|=\sqrt{B_{x}^{2}+B_{y}^{2}+B_{z}^{2}}\right)$ in $x=0$ plane at $t=0 \mathrm{Myr}$ (left) and at $t=5 \mathrm{Myr}$ (right) for the same SFR as in Fig. 1. The initial magnetic field has an exponential distribution (see Equation 1) and in the log scale field lines appear as straight lines with constant slope. The magnetic field in the disc gets advected with the gas resulting in magnetized outflows. Notice the unmagnetized bubbles $(y \sim 2-4 \mathrm{kpc})$ due in injection of gas that does not contain magnetic field. Near the mid-plane, due to a number of injection points (see Section 2.3), a substantial region get depleted of magnetic field and that creates a low-emissivity region close to $z \sim 0$.

and injected gas. Disc gas tracer has been set so that it has high value $(\sim 10)$ in cells which are dominated by disc gas at $t=0$ identified using a density criterion and low $(\sim 0)$ otherwise. We find that in Fig. 3 the regions that correspond to high value of the disc tracer (green patches) have a high magnetic field (orange patches). This is expected because some of the disc gas is lifted up by outflows. The right-hand panel of Fig. 3 shows the plasma$\beta$ parameter, which is the ratio of the thermal pressure $\left(P_{\mathrm{th}}\right)$ and magnetic energy density $\left(\beta=8 \pi P_{\mathrm{th}} /|B|^{2}\right)$. Since the initial magnetic field is small, $\beta$ parameter is greater than unity everywhere, even close to the disc and behind the outer shock, where material gets accumulated. 


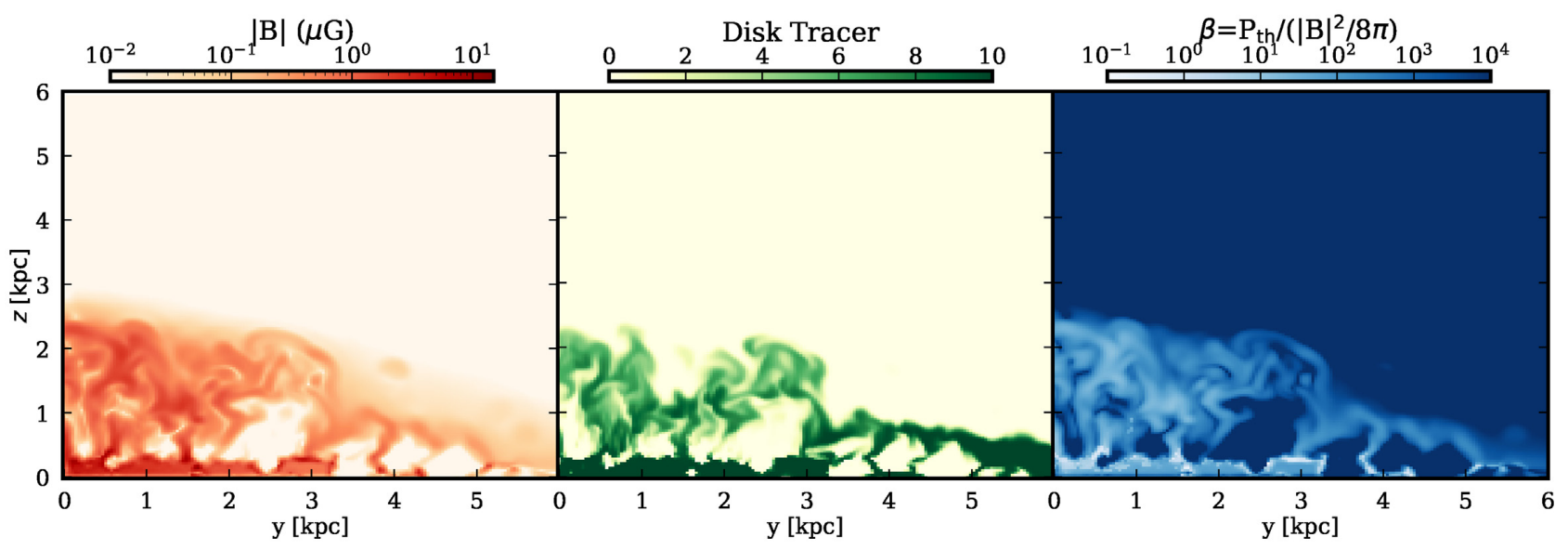

Figure 3. Magnetic field strength, $|B|=\sqrt{B_{x}^{2}+B_{y}^{2}+B_{z}^{2}}$, in $x=0$ plane at $t=10 \mathrm{Myr}$ (left panel) is compared with disc gas tracer (middle panel) in the same plane and the same time. Note that the field strength is high in regions where the disc tracer is high because magnetic field get lifted up along with the disc gas. Plasma $\beta$-parameter $\left(\beta=8 \pi P_{\mathrm{th}} /|B|^{2}\right)$, shown in the right panel, is lower in disc region, where magnetic field is high, and also in the regions where the magnetic field has been compressed along with the gas due to shocks.

\subsection{Synchrotron emission}

CRs are generated through supernova explosions in the disc of the galaxy. In the presence of magnetic field, CREs emit synchrotron radiation. CR particles are believed to get accelerated at shocks and simulations have estimated that they can gain $\sim 10$ per cent of the bulk energy density (Caprioli \& Spitkovsky 2014). Of this, CREs account for a fraction, $\left(m_{e} / m_{p}\right)^{(3-p) / 2}$, depending on the slope of their energy spectrum, $p$ (Persic \& Rephaeli 2014). For $p=2.2$, this fraction is $\sim 5$ per cent. Thus, given gas energy density, $\mathrm{u}_{\text {gas }}, \epsilon_{\mathrm{CRE}}$ $\equiv \mathrm{u}_{\mathrm{CRE}} / \mathrm{u}_{\mathrm{gas}}$ is equal to $0.01 \times 0.05=5 \times 10^{-3}$.

We can write down the emissivity, $j_{v}$, in terms of $\epsilon_{\mathrm{CRE}}$ and the magnitude of magnetic field $(B)$ as (refer to equation 18.17 of Longair 1981)

$$
\begin{aligned}
j_{v}\left(\mathrm{ergs}^{-1} \mathrm{~cm}^{-3} \mathrm{~Hz}^{-1}\right)= & 1.7 \times 10^{-21} \times a(p) \times k \times\left(\epsilon_{\mathrm{CRE}} \times u_{\mathrm{gas}}\right) \\
& \times B^{(p+1) / 2)}\left(\frac{6.26 \times 10^{18}}{v}\right)^{\frac{(p-1)}{2}} .
\end{aligned}
$$

$k$ is the normalization for the energy spectrum, i.e. $\mathrm{u}_{\mathrm{CRE}}=$ $\int_{m_{e} c^{2}}^{\infty} k E^{-(p-1)} \mathrm{d} E . a(2.2) \sim 0.1$, from table 18.1 of Longair (1981). We take $v=1.4 \mathrm{GHz}$ for the rest of our analysis.

\subsubsection{CR energy density}

One has to necessarily resort to assumptions when calculating the synchrotron radio emission from MHD simulations. The objective is to find a proxy for CR energy density for which there are different recipes. We assume $u_{\mathrm{CRE}} \equiv \epsilon_{\mathrm{CRE}} \times \mathrm{u}_{\mathrm{gas}}$ to be proportional to magnetic or gas thermal/kinetic energy density. Since kinetic energy is frame dependent, caution should be exercised while using it for getting $u_{\mathrm{CRE}}$. However, it can be a reasonable proxy if gas motions are turbulent, since internal shocks as well as adiabatic compression can give rise to CRs. In this section, we discuss how these different prescriptions will affect the total radio luminosity.

To understand how these different prescriptions will affect the observables, in Fig. 4 we show the radio power at $1.4 \mathrm{GHz}, P_{v, 1.4 \mathrm{GHz}}$, for the simulation box as a function of time. The total power is obtained by summing up the emissivity (given by equation 4) for each cell in the simulation domain and multiplying with the total volume of the box, $P_{v, 1.4 \mathrm{GHz}}=\int j_{v} \mathrm{~d} V$. We obtain different curves by taking $\mathrm{u}_{\mathrm{CRE}}$ proportional to thermal pressure $\left(=1.5 P_{\mathrm{th}}\right)$, kinetic energy density $\left(=1.5 P_{\text {kin }}, \mathrm{P}_{\text {kin }}=0.5 \rho\left(\mathrm{v}_{x}^{2}+\mathrm{v}_{y}^{2}+\mathrm{v}_{z}^{2}\right)\right)$, and sum of the two. In the left-hand panel, we show total power for $3 \mathrm{M}_{\odot}$ $\mathrm{yr}^{-1}$ run from the entire box. The luminosity according to thermal energy density declines with time which is unphysical, since there is a continuous energy injection from star formation. Further, we note that the kinetic pressure component dominates the thermal pressure component of total power.

In the dashed curve of the left-hand panel of Fig. 4, we show the power if $\mathrm{u}_{\mathrm{CRE}}$ was in equipartition with magnetic field energy density $\left(=|B|^{2} / 8 \pi\right)$. The total power obtained by making this assumption falls short of analytical estimates (see expression 5). This is not surprising since the initial magnetic energy density is not in equipartition with the gas energy density.

The three curves in the right two panels of Fig. 4 show the temporal dependence of radio power for all the SFRs assuming $u_{\mathrm{CRE}} \propto\left(P_{\mathrm{th}}+P_{\text {kin }}\right)$. The middle panel shows the power for the entire simulation domain and the right-hand panel shows the contribution of the extra-planar gas $(|z|>400 \mathrm{pc})$. Power for $30 \mathrm{M}_{\odot} \mathrm{yr}^{-1}$ decreases after about $\sim 5$ Myr once the outer shock moves out of the box and outflows escape the simulation domain. For $3 \mathrm{M}_{\odot} \mathrm{yr}^{-1}$, the power increases monotonically throughout the entire run of 10 Myr. From Figs 1 and 3, we see that the disc contains substantial gas as well as magnetic field intensity even in the presence of outflows. We, therefore, expect the extra-planar component to be sub-dominant (the right-hand panel of Fig. 4). Once the outflows breach the scale height of the disc (after about 2 Myr for 3 and $30 \mathrm{M}_{\odot} \mathrm{yr}^{-1}$ ), the power emitted remains nearly a constant. In the following sections, we discuss how radio power depends on time as well as the magnetic field and SFR.

In order to select an appropriate measure of $u_{\mathrm{CRE}}$, we can compare the corresponding radio power with one-zone model. We can estimate a conservative value of the synchrotron luminosity, by taking $\mathrm{u}_{\text {gas }}$ equal to $L_{\text {mech }} \times t$. $\mathrm{P}_{\nu, 1.4 \mathrm{GHz}}$ can be written as

$$
\begin{aligned}
P_{v, 1.4 \mathrm{GHz}} \approx & 4 \times 10^{26} \mathrm{erg} \mathrm{s}^{-1} \mathrm{~Hz}^{-1}\left(\frac{t}{10 \mathrm{Myr}}\right) \\
& \times\left(\frac{L_{\mathrm{mech}}}{3 \times 10^{41} \mathrm{erg} \mathrm{s}^{-1}}\right)\left(\frac{B}{\mu \mathrm{G}}\right)^{1.6} .
\end{aligned}
$$




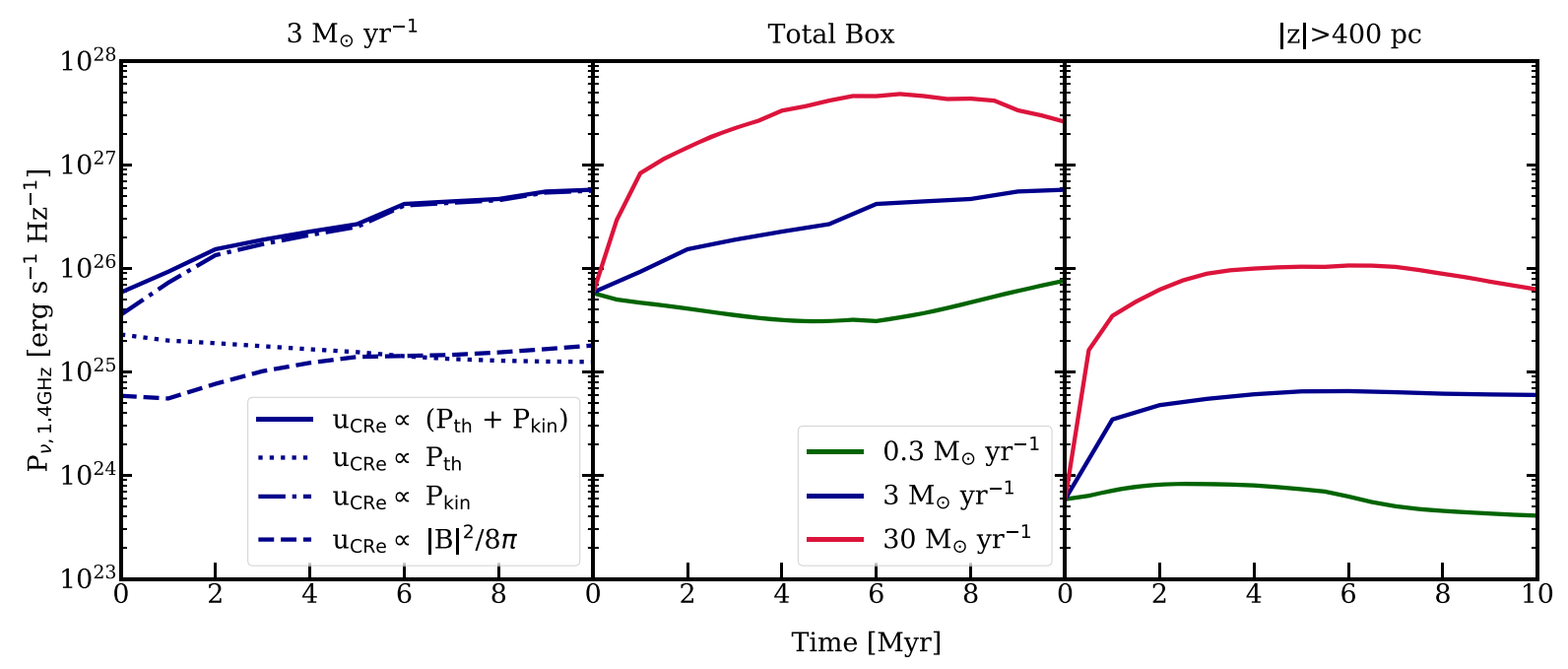

Figure 4. Evolution of total synchrotron power emitted at $1.4 \mathrm{GHz}$ for different expressions for $\mathrm{u}_{\mathrm{CRE}}$ (left) and SFRs (middle and left). The left-hand panel shows the power emitted by the entire simulation domain for $3 \mathrm{M}_{\odot} \mathrm{yr}^{-1}$ case by using $\mathrm{u}_{\mathrm{CRE}}$ proportional to thermal energy density $\left(P_{\mathrm{th}}\right.$, dotted line), kinetic energy density $\left(P_{\text {kin }}, P_{\text {kin }}=0.5\left(v_{x}^{2}+v_{y}^{2}+v_{z}^{2}\right)\right.$, dot-dashed line), total gas energy density $\left(P_{\text {kin }}+P_{\text {th }}\right.$, solid line), and magnetic energy density $\left(|B|^{2} / 8 \pi\right.$, dashed line) (see equation 4). The total power estimated using the sum of thermal and kinetic energy gives a number closest to analytical estimates (see expression for total power, $P_{v}$, relation 5). Thus, we use it to find the power for other SFRs - shown in the two right-hand panels. The middle panels shows total power from the entire simulation domain for three SFRs -0.3 (green), 3 (blue), and 30 (red) curves. The red curves increases and then shows a decrease after $\sim 5$ Myr once the outer shock moves out of the simulation domain. The green curve shows the exact opposite trend because of intense radiative losses in the disc before the outer shock can break out of the disc. The right-hand panel shows the power emitted by the extra-planar region, which attains a steady-state value within 2-3 Myr. The green curve in this panel-hand hardly changes from its $t=0$ value, thus exhibiting very low extended emission.

At $10 \mathrm{Myr}$, the power estimated from using the sum of thermal and kinetic energy density gives $\approx 3 \times 10^{26} \mathrm{erg} \mathrm{s}^{-1}$, while the estimated power is $\approx 4 \times 10^{26}$, for $B \simeq 1 \mu \mathrm{G}$.

Comparison with the curves in Fig. 4 (left-hand panel) shows that using only the thermal energy density leads to an underestimate of the luminosity, and also produces a declining luminosity over time, contrary to the expectations from equation (5). Using the sum of thermal and kinetic energy density provides a better match with the observed luminosity. The volume-averaged magnetic field within the disc, dominant source of synchrotron emission, is a decreasing function of time. This is because the injected gas evacuates larger and larger un-magnetized holes. Therefore, the radio luminosity would not grow linearly in time, as seen from the green and blue curves in Fig. 4. We also note that the estimate of the radio luminosity in equation (5) matches the observed values (e.g. refer to table 3 of Dahlem et al. 1995). We need to scale the power by surface density of energy injection rate, $\dot{E}_{A}$, the radius of star formation, $r_{\mathrm{SF}}$, equal to $4 \mathrm{kpc}$ for our simulations, and the magnetic field. We get $P_{v} \sim \dot{E}_{A} r_{\mathrm{SF}}^{2} B^{1.6}$ and for NGC $4666\left(r_{\mathrm{SF}}=13 \mathrm{kpc}, B=8.8 \mu \mathrm{G}\right)$ this comes out to be $\sim 5 \times 10^{26} \mathrm{erg} \mathrm{s}^{-1}$, comparable to the power at $10 \mathrm{Myr}$ for $3 \mathrm{M}_{\odot} \mathrm{yr}^{-1}$.

Therefore, we adopt the sum of kinetic and thermal energy densities as the gas energy density and use it for synchrotron emissivity for further analysis. We note here that the conclusion - that kinetic energy determines synchrotron power - follows from the fact that magnetic field energy density is much lower than the gas thermal energy density (see the right-hand panel of Fig. 3). Observationally, it has been found that $u_{\mathrm{CRE}}$ and magnetic field energy density are in equipartition (Beck \& Krause 2005; Beck 2012)

Using equation (4) for emissivity, we can produce surface brightness maps for the outflows. Surface brightness is obtained by multiplying emissivity and the elemental length along the line of sight, taken to be $x$-axis. In Fig. 5, we show the surface brightness at $5 \mathrm{Myr}$ for different SFRs, increasing from left to right. Due to the presence of strong magnetic field, the disc is the strongest emitter of synchrotron radiation. The emission decreases in regions above and below the disc as the injected gas pushes on the disc gas and fills the volume within the outer shock. At much higher heights, $z \sim 4-5 \mathrm{kpc}$, the material collects in the turbulent region behind the outer shock. There is insignificant extra-planar emission for the lowest SFR but its extent increases with SFR. This results in increased emission at this height, which we refer to as the 'bump'. The observational consequences of the bump are discussed in Section 3.2. One can compare the simulated surface brightness map in Fig. 5 with the high-resolution images of edge-on starburst galaxies. For example that of NGC 4666 (see fig. 6 in Irwin et al. 1999), which shows filamentary structure because of chimneys created during the breakout of gaseous outflow from the disc. Such a filamentary structure, extending outwards from the disc, is visible in two right-hand panels of Fig. 5.

In Fig. 6, we show the scatter plot between the density and magnitude of magnetic field for $3 \mathrm{M}_{\odot} \mathrm{yr}^{-1}$ case at $5 \mathrm{Myr}$. The plot has been colour-coded by synchrotron power. We separate the simulation domain into disc (right-hand panel) and extra-planar region (left-hand panel). The dot-dashed lines in both the plot have a slope of $\rho^{2 / 3}$. The distribution of high emissivity cells in the disc region follows closely the $\rho^{2 / 3}$ slope. In the extra-planar regions, we see adherence to the relation especially as extension in the highdensity region of the plot. It should be noted that in the extra-planar region, the disc gas has been mixed with the fresh injected gas that carries no magnetic field. We therefore do not expect the relation, arising out of flux freezing, to apply very strongly in $z>400 \mathrm{pc}$ region. 


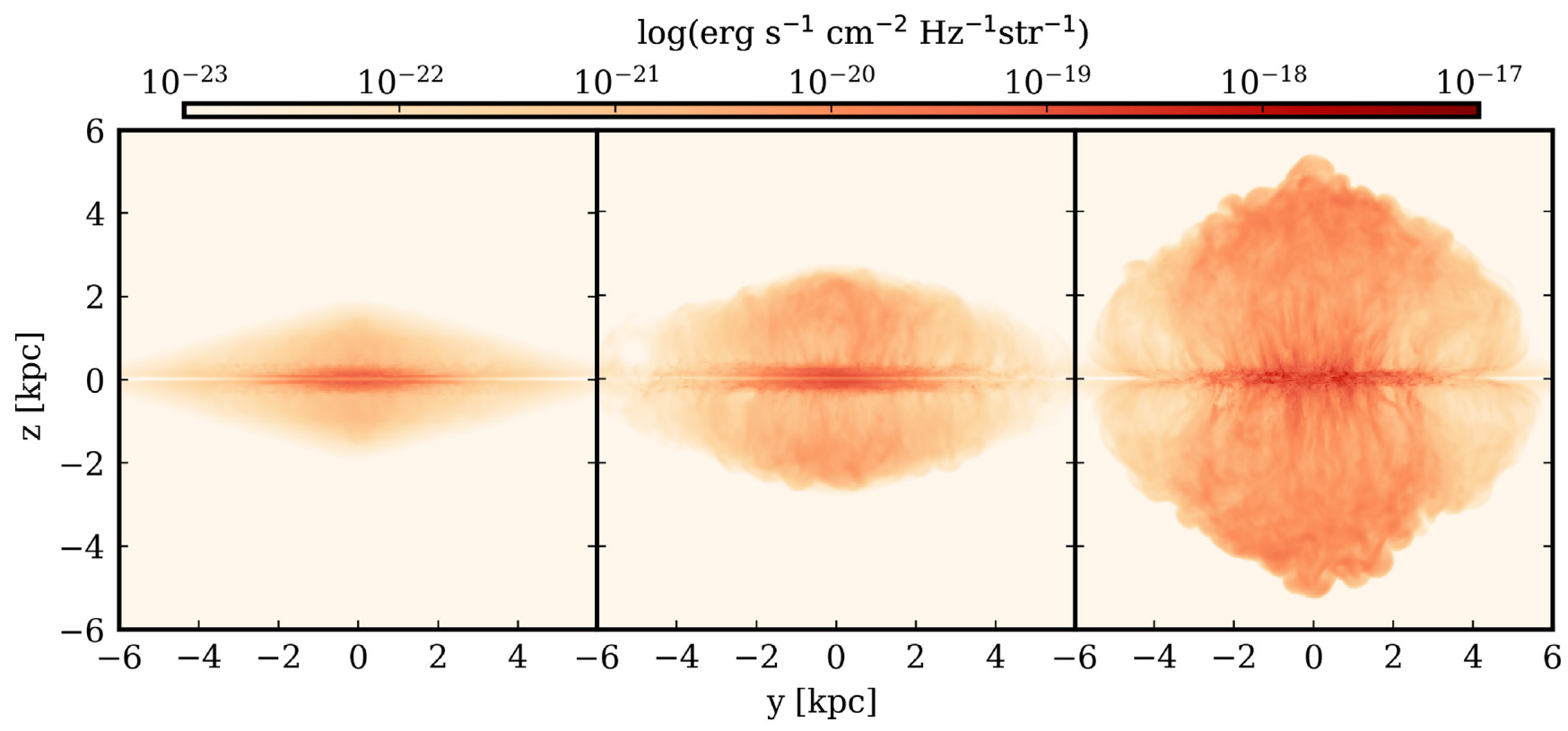

Figure 5. Surface brightness snapshots at $5 \mathrm{Myr}$ by taking $u_{\text {gas }} \propto\left(P_{\text {th }}+P_{\text {kin) }}\right.$ for $\mathrm{SFR}=0.3,3$, and $30 \mathrm{M}_{\odot} \mathrm{yr}^{-1}$ (from left to right). There is hardly any extra-planar emission from $0.3 \mathrm{M}_{\odot} \mathrm{yr}^{-1}$ case even after $5 \mathrm{Myr}$ (compare with the left-hand panel of Fig. 2). Filamentary structure in emission is observed in 3 and $30 \mathrm{M}_{\odot} \mathrm{yr}^{-1}$ cases, as channels are created by outflows from the disc. Enhancement of emission is behind the outer shock as a result of gas accumulation.

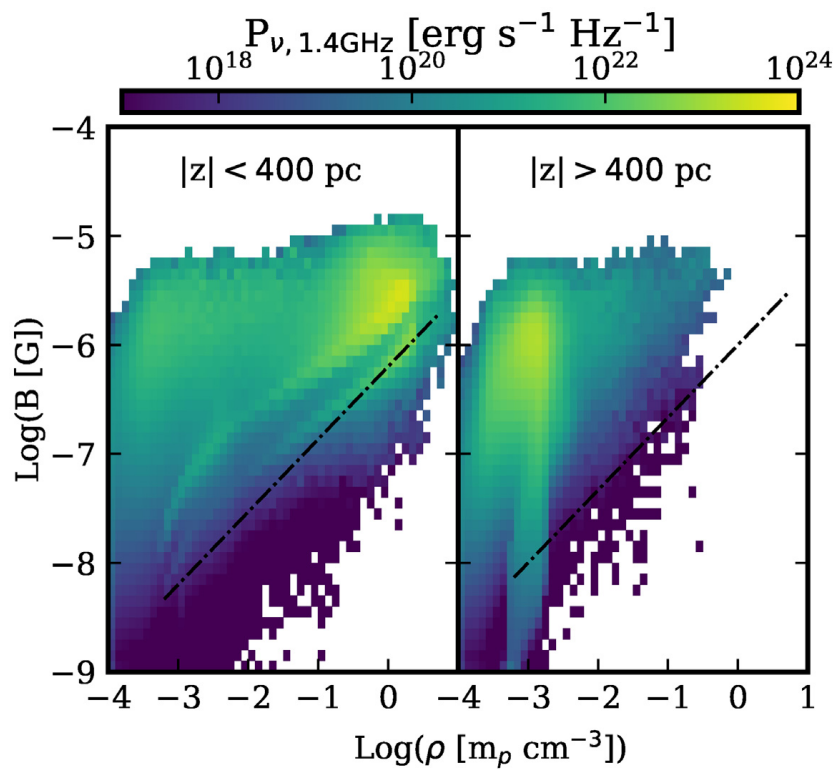

Figure 6. Scatter plot between density, $\rho$ (along horizontal axis), and magnitude of magnetic field, $B$, along vertical axis, colour-coded by synchrotron power for $3 \mathrm{M}_{\odot} \mathrm{yr}^{-1}$ case at $5 \mathrm{Myr}$. The left-hand panel shows this distribution for the disc region $(z<400 \mathrm{pc})$, while the right one is for the extra-planar region $(z>400 \mathrm{pc})$. The dot-dashed line represents power-law relation between $B$ and $\rho, B \propto \rho^{2 / 3}$ (assuming isotropicity), expected from flux freezing. The gas in the disc follows this relation due to flux freezing. The magnetic field strength of the extra-planar gas has been diluted by the injected gas and does not follow this relation very strongly.

\subsection{Scale height of emission}

Fig. 7 shows the vertical intensity profile of radio emission. We obtain vertical intensity profiles by summing the emissivities in the $x-y$ plane in a slab of thickness $36 \mathrm{pc}$ and then plotting it along

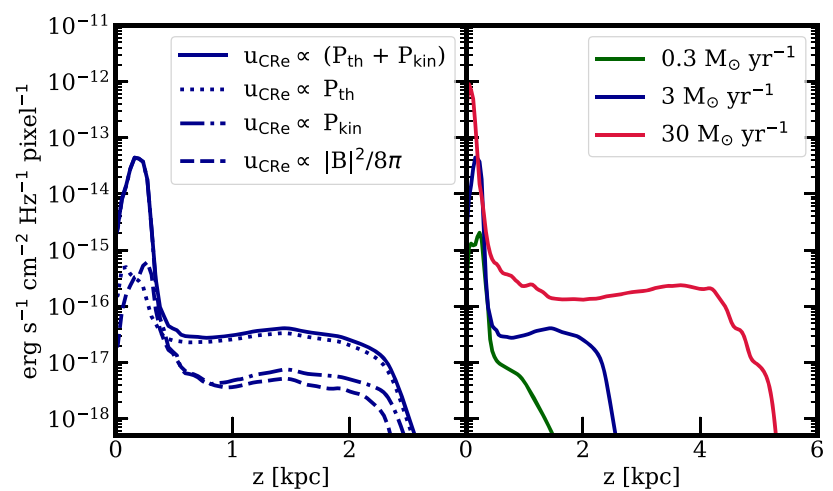

Figure 7. Vertical intensity profiles for $\mathrm{SFR}=0.3,3$, and $30 \mathrm{M}_{\odot} \mathrm{yr}^{-1}$ at $5 \mathrm{Myr}$. In the left-hand panel, the dotted, dot-dashed, dashed, and solid curves have been obtained in the same way as for Fig. 4. We see that near the mid-plane the emission is dominated by kinetic energy, while thermal energy is responsible in the higher $z$ regions. The right-hand panel shows the vertical intensity for the three SFRs $-0.3,3$, and $30 \mathrm{M}_{\odot} \mathrm{yr}^{-1}-$ in green, blue, and red curves. The red and blue curves show a characteristic feature - plateau followed by a slight bump.

the $z$-axis. The curves in the left-hand panel show this profile for SFR of $3 \mathrm{M}_{\odot} \mathrm{yr}^{-1}$ at $5 \mathrm{Myr}$ using different prescriptions for $\mathrm{u}_{\mathrm{CRE}}$. The thermal energy density in the disc is low due to presence of cold gas and the kinetic energy of the rotating disc is expected to dominate. However, as the gas is lifted up to extra-planar height, we see the reverse. The right-hand panel shows the profile for the different SFRs. As visible in surface brightness plots, the extent of emission increases with SFR. The bump feature mentioned in the previous section is clearly seen in vertical profiles, especially in the red curve.

In order to compare these simulated vertical intensity profile images with observed images, one has to convolve the simulated images with typical beam sizes of radio telescopes. The vertical 


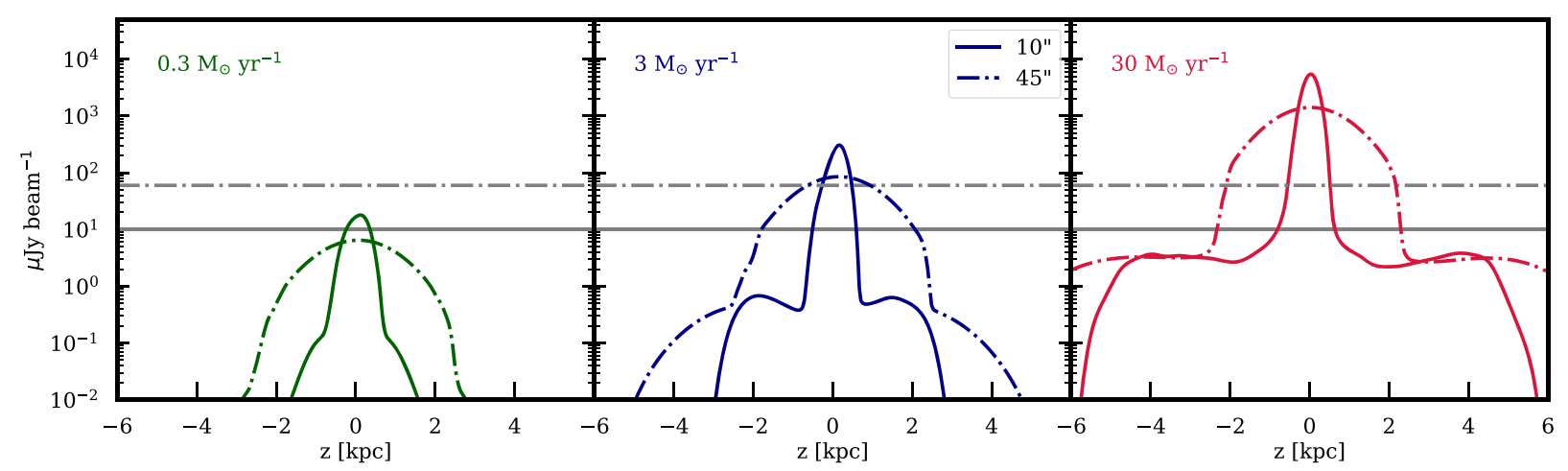

Figure 8. Vertical intensity profiles for different SFRs and different choices for $u_{\mathrm{gas}}$ (see Section 3.1.1) $0.3 \mathrm{M}_{\odot} \mathrm{yr}^{-1}$ (left), $3 \mathrm{M}_{\odot} \mathrm{yr}^{-1}$ (middle), and $30 \mathrm{M}_{\odot}$ $\mathrm{yr}^{-1}$ (right) convolved with beams of FWHM 10 arcsec (solid) and $45 \operatorname{arcsec}$ (dot-dahsed). The horizontal lines show the rms noise levels at $10 \mu \mathrm{Jy}$ (solid) and $60 \mu \mathrm{Jy}$ (dot-dashed). We assume that the galaxy is at a distance of $10 \mathrm{Mpc}$ from Milky Way. The plateau feature is undetectable even for the highest star formation we have used.

intensity profile $I_{v}(z)$ is convolved with a Gaussian of width $\sigma$ to yield the convolved profile:

$I_{\nu, \text { convolved }}\left(z_{1}\right)=\frac{\int I_{v}(z) \exp \left[-\frac{\left(z-z_{1}\right)^{2}}{2 \sigma^{2}}\right] \mathrm{d} z}{\int \exp \left[-\frac{\left(z-z_{1}\right)^{2}}{2 \sigma^{2}}\right] \mathrm{d} z}$,

where $\sigma=\mathrm{FWHM} / 2 \sqrt{2}$, and the full width at half-maximum (FWHM) is obtained by the length subtended by the angle of the beam at the distance of the galaxy. For this purpose, we take the example of a well known and nearby edge-on star-forming galaxy, NGC 891, which is also a Milky Way-type galaxy (rotational speed of $\sim 220 \mathrm{~km} \mathrm{~s}^{-1}$ ), located at a distance of $9.5 \mathrm{Mpc}$ (Fraternali et al. 2011). We assume a distance of $10 \mathrm{Mpc}$ for the simulated galaxy. For a typical radio telescope, we use the parameters of the Giant Meter-Wave Radio Telescope (GMRT), which has a synthesized beam of $10 \operatorname{arcsec}(45 \operatorname{arcsec})$ at $L$ band, with an rms noise per beam of $\sim 10(60) \mu \mathrm{Jy}$ for the entire (compact) array (Dwarakanath private communication). Fig. 8 shows convolved vertical intensity profiles from Fig. 7 for beams with FWHM of 10 arcsec (solid) and 45 arcsec (dot-dashed). The corresponding horizontal lines show the rms noise per beam for GMRT.

The extra-planar emission is unlikely to be detected with either the synthesized beam or with the beam produced by the compact array of GMRT. The emission becomes prominent with increasing SFR. Even for a high SFR, the extra-planar radiation is below the noise level, making it difficult to detect. It is then easy to understand why the observed profiles never reported such an enhanced patch of emission above the gas scale height. It is likely to be detected for very high SFR, for starburst galaxies. The nearest galaxy with a SFR of $\sim 7.7 \mathrm{M}_{\odot} \mathrm{yr}^{-1}$ (Li \& Wang 2013), M82, has never been imaged with attention given to extended low surface brightness features. It will be an interesting test of our result if a broad beam is used in order to detect such features. Such an accumulation of matter has been observed in other wavebands. M82's vertical intensity profile for CO intensity also shows a bump above the gaseous scale height (see fig. 11 of Leroy et al. 2016), similar to the continuum emission plateau plotted in Fig. 8.

It is interesting to note that NGC 4631 (SFR $\sim 1.3 \mathrm{M}_{\odot} \mathrm{yr}^{-1}$, Weigert et al. 2015), an edge-on galaxy has been observed with the Ooty Synthesis Radio Telescope, and its vertical intensity profile shows a plateau of the type we have described above (Sukamar \& Velusamy 1985). Sukamar \& Velusamy (1985) did not observe

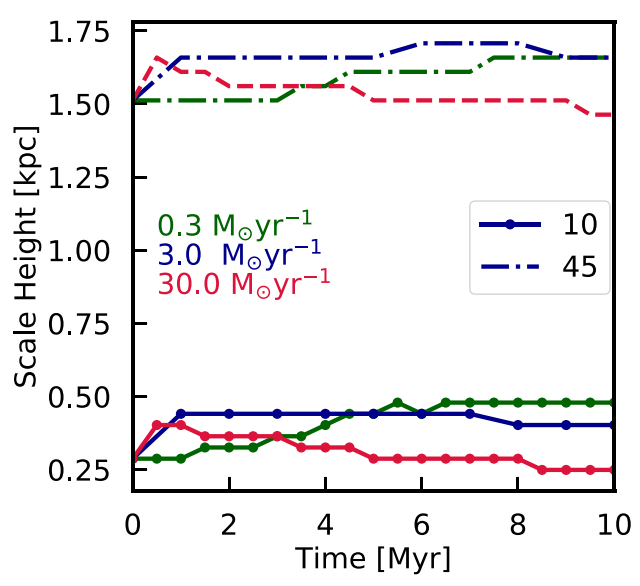

Figure 9. Variation of scale heights with time for $0.3,3$, and $30 \mathrm{M}_{\odot} \mathrm{yr}^{-1}$ (red and blue, respectively). The dashed lines for a resolution of 45 arcsec and dots represent a resolution of 10 arcsec. Scale height has been found using Fig. 7 (see Section 3.2). We see it reflects the beam size.

the plateau at $1.4 \mathrm{GHz}$, possibly because of lower flux density at high frequency. It will be interesting to observe this galaxy again with more sensitive instruments. Since current observations cannot detect the low surface brightness plateau, we use convolved profile in order to estimate the radio scale height.

In Fig. 9, we show how the radio emission scale height changes with time for different SFRs and beam sizes. We have determined the scale height as the height at which the convolved vertical intensity profiles of Fig. 7, fall by a factor of $e$. For a higher resolution (FWHM $\sim 10$ arcsec), the scale height is between $250-500 \mathrm{pc}$ for all the three SFRs, while for a larger beam size it lies between 1.5 and $1.75 \mathrm{kpc}$. The scale heights remain close to the linear resolution represented by beam sizes $(0.5$ and $2.2 \mathrm{kpc}$ on an object at a distance of $10 \mathrm{Mpc}$ for 10 and $45 \mathrm{arcsec}$, respectively). The slight variation in scale heights with time is a result of changing nature of outflows.

In Fig. 10, we show the time-averaged radio emission scale height for three SFRs. Krause et al. (2018) presented detailed results for NGC 5775, which has the same morphological type as MW (although with a higher SFR $\sim 14 \mathrm{M}_{\odot} \mathrm{yr}^{-1}$; Irwin et al. 2012) at a distance of $20 \mathrm{Mpc}$, in the $L$ band of VLA. They use a resolution of 


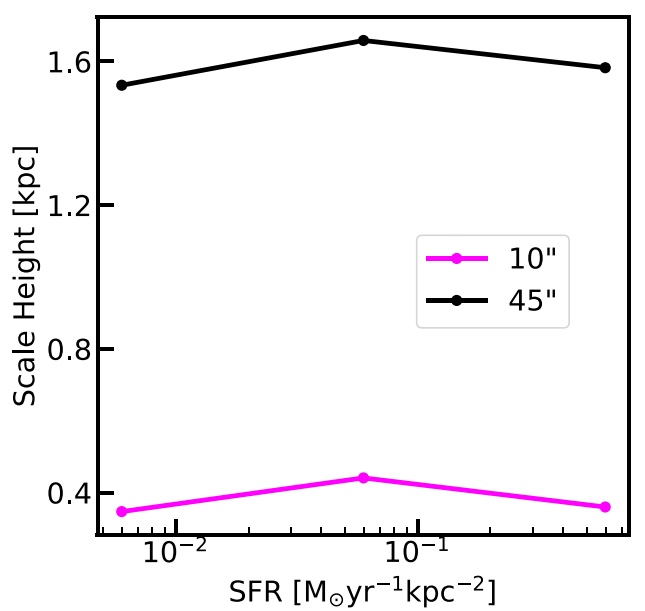

Figure 10. Variation of scale heights with SFR for two different resolutions -10 and 45 arcsec. The scale height is independent of the SFR.

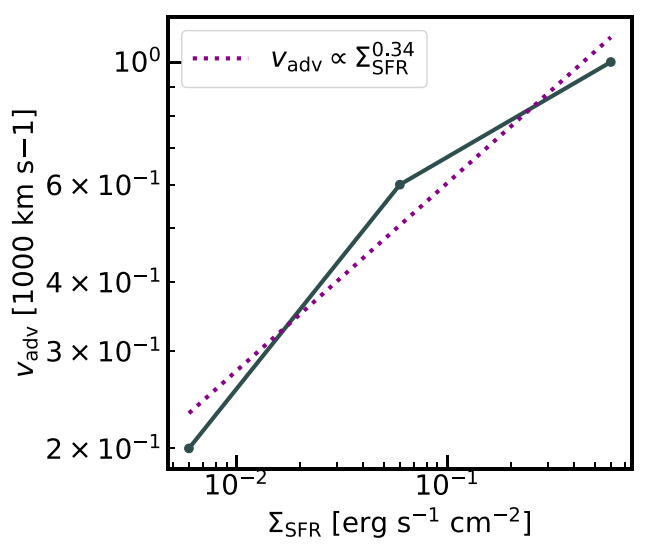

Figure 11. Advection speed ( $v_{\text {adv }}$ ) versus SFR surface density is shown by the blue curve. Advection speed is measured by estimating the distance travelled by the outer shock in 5 Myr. The dotted line shows a power-law fit with $v_{\mathrm{adv}} \propto \Sigma_{\mathrm{SF}}^{1 / 3}$.

10 arcsec and report the $L$-band scale height as $\sim 2 \mathrm{kpc}$. To make a comparison with Fig. 9, we would require an equivalent resolution of $20 \mathrm{arcsec}$, with our fiducial galaxy at $10 \mathrm{Mpc}$. It is therefore reasonable to conclude that the scale height would lie between the magenta and black curves in Fig. 9, and is roughly consistent with the reported scale height.

\subsection{Advection speed}

We show the variation of advection speed of the outer shock with SFR surface density in Fig. 11. The speed is estimated by the distance travelled by the outer shock in $5 \mathrm{Myr}$. The curve shows that the speed scales with SFR surface density roughly as $\mathrm{v}_{\text {adv }} \propto \Sigma_{\mathrm{SFR}}^{1 / 3}$. This is consistent with the observations of Heesen et al. (2016), and can be understood from a similarity analysis of a planar blast wave (Vijayan et al. 2018). Consider the self-similar motion of a $1 \mathrm{D}$ shock, driven by energy injection in the disc through the extra-planar region. The relevant parameters for the evolution of the shock are the halo density $\rho$, the SFR surface density $\Sigma_{\text {SFR }}$ and time $t$. These parameters can be combined to give the height of the shock at a given time as, $R \propto\left(\Sigma_{\mathrm{SFR}} t^{3} / \rho\right)^{1 / 3}$, which gives the speed of the shock as $\mathrm{v}_{\mathrm{adv}} \propto \Sigma_{\mathrm{SFR}}^{1 / 3}$, as observed, and as found in our simulation.

Advection of gas into the extra-planar region in the abovementioned manner has an interesting implication. Once the disturbance due to injection of matter and energy in the disc has been able to break out of the disc, the energy that is being deposited due to star formation is readily transported to the extra-planar region. Consider again a parallel slab geometry, and the time evolution of the gas in the extra-planar region. The above considerations show that the height scales with time as $R \propto t$, which implies (since the radial extent of the extra-planar region does not change with time) that the volume of the extra-planar gas also scales as $V \propto t$. The total energy content of the gas increases linearly with time, since the SFR is assumed to be constant. This means that the gas pressure in the extra-planar region stays roughly constant with time (since volume and total energy both increase linearly with time). Also, the energy content of the CREs increases linearly with time. However, due to flux freezing, the magnetic field in the extra-planar region decreases with volume as $B \propto V^{-2 / 3}$ and therefore, with time as, $B \propto t^{-2 / 3}$. The synchrotron luminosity of the extra-planar gas depends on the total energy content of the CR electrons (which scales as $t$ ) multiplied by $B^{1.6}$ (which scales roughly as $t^{-1}$, because of the above-mentioned scaling). These two factors cancel to give a roughly constant luminosity for the extra-planar gas with time (see Fig. 4)

\section{DISCUSSION}

Our conclusions for the radio scale height have important implications for the shape of the radio halo of star-forming galaxies. Since diffusion time-scale is much longer than the advection timescale on $>\mathrm{kpc}$ scales, the radial and vertical extents of the radio halo are unlikely to be different from as represented in our paper, even in the presence of diffusion. The radial extent of the radio halo is, therefore, determined by the extent of the star-forming regions in the disc, i.e. $4 \mathrm{kpc}$ in our simulation. Therefore, a radio scale height of $0.4-2 \mathrm{kpc}$ implies a ratio of major-to-minor axis of $\sim 2-10$. This is consistent with the findings of Singal et al. (2015) of major-to-minor ratio in edge-on star-forming galaxies of being $\approx 2.5 \pm 1.1$, also similar to a 'flattened ellipsoid' inferred by Weigert et al. (2015) from stacking 30 edge-on spiral galaxies. The near spherical-halo model of Milky Way of Subrahmanyan \& Cowsik (2013), proposed to explain the radio excess of Kogut et al. (2011) without invoking any new population of sources or a cosmological origin, requires, in contrast, a ratio of 1.24. Therefore, our findings point out a discrepancy with the almost-spherical halo model of star forming galaxies and create issues for non-cosmological origin of the radio excess. If we consider diffusion, to make the radio halo more spherical (making the major to minor ratio $1.24 \pm 0.09$ ), the diffusion coefficient would have to be $\gtrsim 10^{29} \mathrm{~cm}^{2} \mathrm{~s}^{-1}$, even for an unrealistic long duration of star formation of $50 \mathrm{Myr}$.

Our results are in agreement with the SFR surface density put forwards by Dahlem et al. (1995) for the existence of radio halo $\left(\Sigma_{\mathrm{SFR}} \gtrsim 10^{-2} \mathrm{erg} \mathrm{s}^{-1} \mathrm{~cm}^{-2}\right)$. For our lowest SFR case $\left(0.3 \mathrm{M}_{\odot}\right.$ $\left.\mathrm{yr}^{-1}\right), \Sigma_{\mathrm{SFR}}$ is equal to $6 \times 10^{-3} \mathrm{M}_{\odot} \mathrm{yr}^{-1} \mathrm{kpc}^{2}$, since star formation sites are confined within $4 \mathrm{kpc}$. The total extra-planar emission from this case is nearly constant with time (the right-hand panel of Fig. 4) and increases as for other cases. We conclude that despite 10 Myr of energy injection, a radio halo could not be sustained for this SFR surface density. However, this threshold is not independent of galaxy mass. It depends on two velocity scales - the advection 
speed, which scale as $\Sigma_{\mathrm{SFR}}^{1 / 3}$ (discussed above) and the escape speed, $\mathrm{v}_{\text {escape }} \propto M^{1 / 3}$, with the galaxy mass $M$. Thus, the threshold energy injection surface density for the existence of radio halo should be smaller for a lower mass galaxy.

A caveat of our simulation is that magnetic field is not in equipartition with the turbulent and thermal energy density, initially. We note that though the differentially rotating gas in the disc will lead to emergence of a large-scale dynamo, this effect is negligible for our simulations. The time period over which this effect is significant is $>1 / \Omega \gtrsim 300 \mathrm{Myr}$, where $\Omega$ is the rotational speed in the disc. Further, we are not injecting any magnetic fields even though supernovae ejecta is expected to be magnetized and supernova-driven superbubbles amplify magnetic fields on similar time-scales (Ferrière \& Schmitt 2000). Therefore, our simulation is inadequate for addressing some aspects of radio emission from the halo at larger scale heights, for example, as observed by HodgesKluck et al. (2018) in the case of NGC 891. Their observation of a higher magnetic pressure than gas pressure at a height of $1<$ $z<3 \mathrm{kpc}$ requires a detailed MHD simulation of the breaking out of magnetized superbubbles, as they have suggested. Our simulation does not take into account halo-scale magnetic field and diffusion of CR particles from the disc as well as their in situ production at large scales, which may affect the prediction of the plateau feature in vertical intensity profile. This work is also different from simulations of CR driven galactic winds (e.g. Dubois et al. 2010; Booth et al. 2013; Pakmor \& Springel 2013), which explicitly include CR pressure in fluid equations, since we rely on gas dynamical processes to estimate the $\mathrm{CR}$ pressure. It is possible that CRs dynamically affect the gas density and pressure profiles, in which case the radio emission characteristics would differ from our estimates. However, some recent studies (e.g. Fujita \& Mac Low 2019) have shown that CRs may not affect galactic outflow structure, which, if confirmed, would make our estimates adequate.

\section{SUMMARY AND CONCLUSIONS}

We conduct 3D MHD simulations of an isolated Milky Way-type galaxy for three different SFR, namely $0.3,3$, and $30 \mathrm{M}_{\odot} \mathrm{yr}^{-1}$. Our initially rotating gas disc comprises a smooth magnetic field (given by equation 1) in the azimuthal direction. Upon comparison with hydrodynamic simulations with identical initial conditions, we see that morphology of outflows are not altered significantly due to magnetic field (Fig. 1). However, the outflowing gas carries magnetic field (see Fig. 2).

Such magnetized outflows lead to presence of radio haloes around star-forming galaxies as a result of synchrotron emission from CREs under the influence of magnetic fields. By assuming that cosmic ray energy density $\left(u_{\mathrm{CRE}}\right)$ is a constant fraction of gas energy density $\left(u_{\text {gas }}\right)$, we can estimate synchrotron emissivity (using equation 4) for all cells in the simulation domain. We explore how different definitions of $u_{\text {gas }}$, such as being equal to thermal pressure $\left(P_{\text {th }}\right)$, kinetic pressure $\left(P_{\text {kin }}\right)$ or sum of both, can affect the macroscopic observables such as total power emitted (Fig. 4) and vertical intensity profiles (Fig. 7). We conclude that using $u_{\text {gas }}$ proportional to the sum of $P_{\text {th }}$ and $P_{\text {kin }}$ can match power with current observations.

Our vertical intensity profiles show a characteristic bump-like feature that is a consequence of accumulation of material behind the outer shock. The profiles for all the SFR show a plateau (see e.g. red curve in Fig. 7 in the vertical range of $z \sim 2-4 \mathrm{kpc}$ for $30 \mathrm{M}_{\odot}$ $\mathrm{yr}^{-1}$ ), and then a slight bump. Such a feature has not been reported in radio observations.

In order to compare our simulation results with the existing data, we produce these profile convolved with appropriate beam size (10 and 45 arcsec). The convolved profiles are shown in Fig. 8. While the convolved profiles faithfully reproduce the plateau plus bump feature, the rms noise levels of instruments (compact array of GMRT) and are unlikely to be detected. This issue can be circumvented for a nearby starburst galaxy, such as NGC 4631. In future, we aim to make observations for this galaxy to test our prediction.

Synchrotron emission has been studied, previously, by measuring the scale height of the radio halo. We use convolved profiles to understand how scale heights vary in time (Fig. 9) and with SFR (Fig. 10). The scale heights depend on the beam size, because the profiles are underresolved.

The main conclusions of our paper are as follows:

(i) Radio haloes are formed as a result of magnetized outflows due to star formation in the disc of a galaxy. Our results of radio halo formed in Milky Way sized are broadly consistent with lower limit of energy injection surface rate density of $\gtrsim 10^{-2} \mathrm{M}_{\odot} \mathrm{yr}^{-1} \mathrm{kpc}^{-2}$ as was observed by Dahlem et al. 1995. We argue that this threshold scales linearly with galaxy mass, explaining the existence of radio halo in smaller galaxies with lower surface SFR densities (see Heesen et al. 2016).

(ii) We see an accumulation of magnetized gas behind the outer shock, resulting in enhanced synchrotron emission from higher $z$ regions of the galaxy. We show that this feature is difficult to observe because of its low surface brightness and extended nature and may have been missed in earlier observation. A notable exception is NGC 4631, which should be revisited in order to test our prediction.

(iii) We find that the scale height of radio halo is independent of SFR, which is consistent with observations.

(iv) We find that the major-to-minor axial ratio is $\sim 2-10$, which points to existence of an oblate radio halo for a star-forming Milky Way mass galaxy. Spherical haloes around the Galaxy have been cited to explain radio excess observed at the Galactic poles.

(v) The advection speed of the radio emitting outflowing gas scaled with SFR as $v_{\text {adv }} \propto \Sigma_{\text {SFR }}^{1 / 3}$. Such a scaling relation has been observed previously (e.g. Heesen et al. 2018).

\section{ACKNOWLEDGEMENTS}

We would like to thank Bhargav Vaidya and Dwarakanath for suggestions and inputs. We would also like to thank the anonymous referee for inputs and suggestions. Some of the simulations discussed in this paper were conducted on the SahasraT cluster at the Super-computer Education and Research Centre, Indian Institute of Science. The work of YS is done under partial support from the joint RFBR-DST project (17-52-45053), by the project 01-2018 'New Scientific Groups LPI', and the Program of the Presidium of RAS (project code 28).

\section{REFERENCES}

Beck R., 2009, ASTRA, 5, 43

Beck R., 2012, SSRv, 166, 215

Beck R., Krause M., 2005, AN, 326, 6

Biermann P. L., Nath B. B., Caramete L. I., Harms B. C., Stanev T., Becker T.-J., 2014, MNRAS, 449, 3879

Booth C. M., Agertz O., Kravtsov A. V., Gnedin N. Y., 2013, ApJ, 777, L16 Caprioli D., Spitkovsky A., 2014, ApJ, 783, 17 
Condon J. J., 1992, ARAA, 30, 575

Cooper J. L., Bicknell G. V., Sutherland R. S., Bland-Hawthorn J., 2008, ApJ, 674, 157

Cooper J. L., Bicknell G. V., Sutherland R. S., Bland-Hawthorn J., 2009, ApJ, 703, 330

Costain C. H., 1960, MNRAS, 120, 248

Dahlem M., Lisenfeld U., Golla G., 1995, ApJ, 441, 119

Dahlem M., Lisenfeld U., Rossa J., 2006, A\&A, 457, 121

Dekel A., Sarkar K. C., Jiang F., Bournaud F., Krumholz M. R., Ceverino D., Primack J. R., 2019, MNRAS, 488, 4753

Dubois Y., Teyssier R., 2010, A\&A, 523, A72

Duric N., Irwin J., Bloemen H., 1998, A\&A, 457, 121

Ferrière K., Schmitt D., 2000, A\&A, 358, 125F

Fixsen D. J. et al., 2011, ApJ, 734, 1

Fraternali F., Sancisi R., Kamphuis P., 2011, A\&A, 531, A64

Fujita A., Mac Low M.-M., 2019, MNRAS, 477, 531

Heesen V. et al., 2018, MNRAS, 476, 158

Heesen V., Dettmar R.-J., Krause M., Beck R., Stein Y., 2016, MNRAS, 458,332

Hodges-Kluck E. J., Bregman J. N., Li J., 2018, ApJ, 866, 126

Hummel E., van der Hulst J. M., 1989, A\&ASS, 81, 51

Hummel E., Dahlem M., van der Hulst J. M., Sukumar S., 1991, A\&A, 246, 10

Irwin J. A. et al., 2012, AJ, 144, 2

Irwin J. A., English J., Sorathia B., 1999, AJ, 117, 2102

Irwin J. A., Saikia D. J., English G., 2000, AJ, 119, 1592

Kogut A. et al., 2011, ApJ, 734, 1

Krause M. et al., 2018, A\&A, 611, 15

Krumholz M. R., Dekel A., McKee C. F., 2012, ApJ, 745, 69

Lehnert M., Heckman T. M., 1996, ApJ, 472, 546

Leroy A. et al., 2016, ApJ, 814, 83

Li J., Wang Q. D., 2013, MNRAS, 428, 2085

Lim B. et al., 2018, MNRAS, 477, 1993L

Longair M. S., 1981, High Energy Astrophysics. Cambridge University Press, Cambridge, NY

Mignone A., Bodo G., Massaglia S., Matsakos T., Tesileanu O., Zanni C., Ferrari A., 2007, ApJSS, 170, 228

Oppenheimer B. D., Davé R., 2006, MNRAS, 373, 1265

Pakmor R., Springel V., 2013, MNRAS, 432, 176

Pakmor R., Marinacci F., Springel V., 2014, ApJ, 783, L20

Persic M., Rephaeli Y., 2014, preprint (arXiv:1405.3107)

Roy A., Nath B. B., Sharma P., Shchekinov Y., 2016, MNRAS, 463, 2296

Roy A., Nath B. B., Sharma P., Shchekinov Y., 2016, MNRAS, 434, 3572

Ruszkowski M., Yang H.-Y. K., Zweibel E., 2016, ApJ, 834, 208R

Salem M., Bryan G. L., 2014, MNRAS, 437, 3312

Singal J., Kogut A., Jones E., Dunlap H., 2015, ApJ, 799, L10

Strickland D. K., Heckman T. M., 2007, ApJ, 658, 258

Subrahmanyan R., Cowsik R., 2013, ApJ, 776, 1
Suchkov A. A., Berman V. G., Heckman T. M., Balsara D. S., 1996, ApJ, 463,528

Sukumar S., Velusamy T., 1985, MNRAS, 212, 637

Sun X. H., Reich W., Waelkens A., Enßlin T. A., 2008, A\&A, 477, 573

Tumlinson J., Peeples M. S., Werk J. K., 2017, ARA\&A, 55, 389

Vasiliev E. O., Shchekinov Y., Nath B. B., 2019, MNRAS, 486, 3685

Vijayan A., Sarkar K., Nath B., Sharma P., Shchekinov Y., 2018, MNRAS, 475,5513

Wiegert T. et al., 2015, ApJ, 153, 202

\section{APPENDIX: CONVERGENCE TEST}

We conduct convergence tests for our simulations. We use resolutions of $20 \mathrm{pc}$ and compare it with our fiducial run at $36 \mathrm{pc}$ $\left(\mathrm{SFR}=3 \mathrm{M}_{\odot} \mathrm{yr}^{-1}\right)$. We conduct the higher resolution with identical initial conditions, including the locations of OB associations. Since the high-resolution run is computationally expensive, we limit the simulation domain of this run to $[-3,3]^{3} \mathrm{kpc}^{3}$. We compare the results from this run with a box of identical dimensions of the lower resolution run.

In Fig. A1, we show density (top) and magnetic field magnitude (bottom) slices in $x=0$ plane for the two resolutions. The general morphology in both the runs is similar for density as well magnetic field magnitude slices. From the density (top) panels, we note that the outer shock has reached a height of $z \sim 3 \mathrm{kpc}$ in $5 \mathrm{Myr}$ for both the cases. We note that a higher resolution run has sharper contact discontinuity at about $2 \mathrm{kpc}$.

In Fig. A2, we compare the total synchrotron power emitted as a function of time upto $5 \mathrm{Myr}$, after which the outer shock moves out of the simulation domain for the higher resolution run. The left-hand panel shows the total power emitted at $1.4 \mathrm{GHz}$ in the entire simulation domain. In the right-hand panel, we show the power for the entire simulation domain for $20 \mathrm{pc}$ (blue) run and $[-3,3]^{3} \mathrm{kpc}^{3}$ box for $36 \mathrm{pc}$ run (red). We see that power for the two resolutions matches closely in the left-hand panel. In the righthand panel, we show total power from extra-planar gas. There is greater difference between power ( $\sim 10$ per cent). From Fig. 7 , we know that thermal energy density dominates in the extra-planar region over the kinetic energy. Because of higher resolution, in the extra-planar region the mixing between the cold gas and the hot gas is efficient, which raises the average temperature of the gas.

From Figs A1 and A2, we conclude that our simulations have converged. 


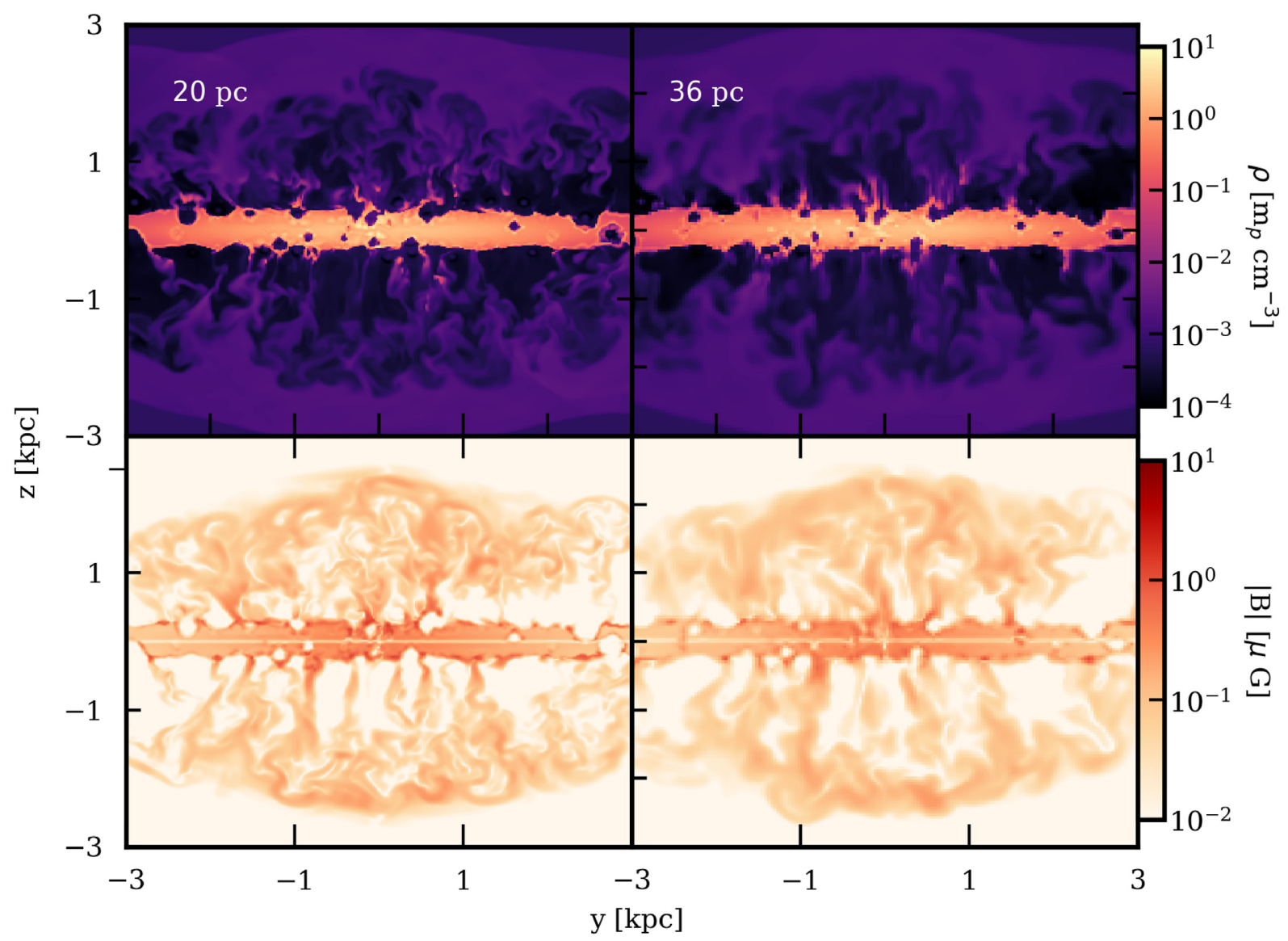

Figure A1. Slices in the $x=0$ plane showing density (top) and magnitude of magnetic field, $|B|=\sqrt{B_{x}^{2}+B_{y}^{2}+B_{z}^{2}}$, (bottom) at 5 Myr. The right-hand panel shows the results from simulations with resolution $20 \mathrm{pc}$, while the left one is from $36 \mathrm{pc}$, which corresponds to the fiducial run. Both the slices have similar morphology.

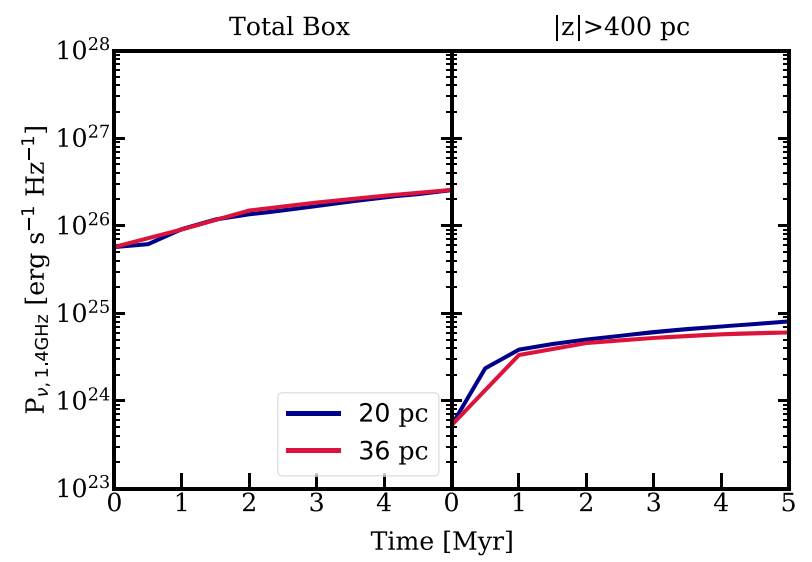

Figure A2. Evolution of total synchrotron power for $\mathrm{SFR}=3 \mathrm{M}_{\odot} \mathrm{yr}^{-1}$ at two different resolutions $-36 \mathrm{pc}$ (blue cuvre) and $50 \mathrm{pc}$ (red). The left-hand panel shows the power emitted by the entire simulation domain. The right shows the power emitted by the extra-planar region.

This paper has been typeset from a $\mathrm{T}_{\mathrm{E}} \mathrm{X} / \mathrm{L} \mathrm{T} \mathrm{E} \mathrm{X}$ file prepared by the author. 